\title{
AN AREA THEOREM FOR HOLOMORPHIC FUNCTIONS
}

\author{
SHINJI YAMASHITA
}

\begin{abstract}
Let $D \varsubsetneqq \mathbf{C}$ and $\Delta \varsubsetneqq \mathbf{C}$ be open sets in the plane $\mathbf{C}$ containing 0 . Suppose that $D$ is connected and $f$ is holomorphic in $D$ with $f(0)=0$. Then, for $Q=f(D) \cap \Delta$ and $P=f^{-1}(Q)$, we have

$$
A(P)+A(Q) \geqslant \pi[\min \{\operatorname{dis}(\partial D, 0), \operatorname{dis}(\partial \Delta, 0)\}]^{2},
$$

$A(\cdot)$ denoting the area. The constant $\pi$ is sharp and the equality holds if and only if $f \equiv 0, \operatorname{dis}(\partial D, 0) \leqslant \operatorname{dis}(\partial \Delta, 0)$, and $D$ is a disk of center 0 .
\end{abstract}

Let $\mathbf{C}$ be the complex plane and suppose that $0 \in D \varsubsetneqq \mathbf{C}$ and $0 \in \Delta \varsubsetneqq \mathbf{C}$, where $D$ is a domain and $\Delta$ is an open set. Then, both $r(D)=\operatorname{dis}(\partial D, 0)$ and $r(\Delta)=$ $\operatorname{dis}(\partial \Delta, 0)$ are positive and finite. Set $r(D, \Delta)=\min (r(D), r(\Delta))$. For $f$ holomorphic in $D$ with $f(0)=0$, write $f^{-1}(\Delta)=\{z \in D ; f(z) \in \Delta\}$. The area of a set $E \subset \mathbf{C}$ is denoted by $A(E)$. We shall prove

THEOREM. We have:

(I) $A(f(D) \cap \Delta)+A\left(f^{-1}(\Delta)\right) \geqslant \pi r(D, \Delta)^{2}$.

(II) The constant $\pi$ in (I) cannot be replaced by any strictly larger one.

(III) The equality in (I) holds if and only if the following hold at the same time:

(III1) $f \equiv 0$,

(III2) $D$ is a disk of center 0 , and

(III3) $r(D) \leqslant r(\Delta)$.

The special case where $D=\Delta=U \equiv\{|z|<1\}$ and $f$ is univalent, is considered by $\mathbf{J}$. L. Ullman in his paper [5, Theorem]; apparently the inequality in (I) is strict in this case, although Ullman does not point this out. Considerable changes must be added to Ullman's skillful method, so that our theorem might be of significance.

For the proof of our theorem we denote $Q=f(D) \cap \Delta$ and $P=f^{-1}(\Delta)$. Then $P=f^{-1}(Q)$.

For the proof of (II) we consider $f(z)=\varepsilon^{1 / 2} z, 0<\varepsilon<1$, for $D=\Delta=U$. Then $A(P)+A(Q)=(1+\varepsilon) \pi$. For the proof of the "if" part of (III), we note that $P=D$ and $A(Q)=0$. Thus,

$$
\pi r(D)^{2}=A(P) \geqslant \pi r(D, \Delta)^{2}=\pi r(D)^{2},
$$

whence the equality in (I) holds.

Received by the editors April 2, 1984 and, in revised form, July 9, 1984.

1980 Mathematics Subject Classification. Primary 30C99.

Kev words and phrases. Areas of the image and the preimage, Alexander-Osserman-Taylor-Ullman's inequality, inner functions, Hardy class $H^{2}$, Frostman's theorem. 
Suppose that $f \equiv 0$. Then $P=D$ and $A(Q)=0$, so that

$$
A(P)+A(Q)=A(D) \geqslant \pi r(D)^{2} \geqslant \pi r(D, \Delta)^{2},
$$

because $D \supset\{|z|<r(D)\}$. Therefore (I) is obtained. Suppose further that in this case, the equality in (I) holds. Then, $A(D)=\pi r(D)^{2}=\pi r(D, \Delta)^{2}$, so that $D=\{|z|$ $<r(D)\}$ and $r(D) \leqslant r(\Delta)$, whence (III2) and (III3) follow.

It remains to be proven that for each nonconstant $f$, (I) holds and the equality in (I) never holds. We need the Alexander-Osserman-Taylor-Ullman inequality [2, Theorem B, p. 767]; [1] in its specified form.

Lemma. Let $F$ be holomorphic in $U$ with $F(0)=0$. If $A(F(U))<\infty$, then $F \in H^{2}$, the Hardy class [3], with

$$
\|F\|_{2}^{2} \leqslant \pi^{-1} A(F(U)),
$$

where

$$
\|F\|_{2}=\left[\lim _{t \rightarrow 1-0}(2 \pi)^{-1} \int_{|\zeta|=1}|F(t \zeta)|^{2}|d \zeta|\right]^{1 / 2}
$$

is the $H^{2}$ norm of $F$. The equality in (1) holds if and only if $F$ is an inner function [3,p. 24] multiplied by a nonnegative constant.

The norm is also expressed by

$$
\|F\|_{2}=\left[(2 \pi)^{-1} \int_{|\zeta|=1}|F(\zeta)|^{2}|d \zeta|\right]^{1 / 2}
$$

where $F(\zeta)=\lim _{t \rightarrow 1-0} F(t \zeta)$ exists at a.e. $\zeta$ on $\partial U$.

Returning to the proof of (I) for nonconstant $f$ we may assume that $A(P)<\infty$ and $A(Q)<\infty$. Let $B$ be the component of the open set $P$ containing 0 . Since $A(B)<\infty$ by $B \subset P$, there exists a universal covering map $g: U \rightarrow B$ such that $g(0)=0$. Then, $h \equiv f \circ g: U \rightarrow f(B)(\subset Q)$ is a holomorphic function.

By our lemma,

$$
\pi\|g\|_{2}^{2} \leqslant A(B) \leqslant A(P)<\infty
$$

and

$$
\pi\|h\|_{2}^{2} \leqslant A(f(B)) \leqslant A(Q)<\infty,
$$

whence

$$
\pi\left(\|g\|_{2}^{2}+\|h\|_{2}^{2}\right) \leqslant A(P)+A(Q) .
$$

Since $g \in H^{2}$ and $h \in H^{2}$, the linear measure $L(\Gamma)$ of

$$
\Gamma=\{\zeta \in \partial U ; g(\zeta) \neq \infty \neq h(\zeta)\}
$$

is $2 \pi$. Set

$$
E_{g}=\{\zeta \in \Gamma ;|g(\zeta)| \geqslant r(D)\}
$$

and

$$
E_{h}=\{\zeta \in \Gamma ;|h(\zeta)| \geqslant r(\Delta)\}
$$


Then,

$$
2 \pi\|g\|_{2}^{2} \geqslant r(D)^{2} L\left(E_{g}\right)
$$

and

$$
2 \pi\|h\|_{2}^{2} \geqslant r(\Delta)^{2} L\left(E_{h}\right) .
$$

If we establish

$$
L\left(E_{g}\right)+L\left(E_{h}\right) \geqslant 2 \pi,
$$

then (I) follows from (4), (5), (6) and (7).

For the proof of (7) it suffices to show

$$
\Gamma=E_{g} \cup E_{h} .
$$

Suppose that there exists $\zeta \in \Gamma \backslash\left(E_{g} \cup E_{h}\right)$. Then, $g(\zeta) \in D$ by $|g(\zeta)|<r(D)$ and $h(\zeta) \in \Delta$ by $|h(\zeta)|<r(\Delta)$. By the continuity of $f$ at $g(\zeta)$, we have $h(\zeta)=$ $\lim _{t \rightarrow 1-0} h(t \zeta)=\lim _{t \rightarrow 1-0} f(g(t \zeta))=f(g(\zeta))$. Therefore $g(\zeta) \in B$ by the definition of $B$ because $g(\zeta)$ is connected with 0 by the curve $\{g(t \zeta) ; 0 \leqslant t \leqslant 1\}$ in $D$. However, this contradicts the covering property of $U$ induced by $g$ over $B$. Thus, (8) is established.

Suppose that the equality in (I) holds for our nonconstant $f$. Since the equalities in (2) and (3) then hold, it follows from our Lemma that there are constants $\alpha \geqslant 0$ and $\beta \geqslant 0$ such that $g=\alpha G$ and $h=\beta H$, where $G$ and $H$ are inner functions; note that $g$ and $h$ are nonconstant. Thus, $|g(\zeta)|=\alpha>0$ and $|h(\zeta)|=\beta>0$ for a.e. $\zeta \in \partial U$.

Suppose that $\alpha<r(D)$. Then $L\left(E_{g}\right)=0$, so that $L\left(E_{h}\right)=2 \pi$ because the equality in (7) holds. Thus, $\beta \geqslant r(\Delta)$. By O. Frostman's celebrated theorem [4, p. 111], $U \backslash H(U)$ is of capacity zero, whence $A(H(U))=\pi$. Therefore,

$$
A(f(B))=A(h(U))=\pi \beta^{2} \geqslant \pi r(\Delta)^{2} \geqslant \pi r(D, \Delta)^{2}=A(P)+A(Q),
$$

so that $A(f(B))>A(Q)$ by $A(P)>0$, which contradicts $f(B) \subset Q$.

Suppose that $\alpha \geqslant r(D)$. Then, by the similar argument for $g$, we have $A(B)=\pi \alpha^{2}$ $\geqslant \pi r(D)^{2} \geqslant A(P)+A(Q)$. Again, $A(B)>A(P)$ by $A(Q)>0$, contradicting $B \subset$ $P$.

\section{REFERENCES}

1. H. Alexander, B. A. Taylor and J. L. Ullman, Areas of projections of analytic sets, Invent. Math. 16 (1972), 335-341.

2. H. Alexander and R. Osserman, Area bounds for various classes of surfaces, Amer. J. Math. 97 (1975), 753-769.

3. P. L. Duren, Theory of $H^{p}$ spaces, Academic Press, New York and London, 1970.

4. O. Frostman, Potentiel d'équilibre et capacité des ensembles avec quelques applications à la théorie des fonctions, Medd. Lunds Univ. Mat. Sem. 3 (1935), 1-118.

5. J. L. Ullman, An area theorem for schlicht functions, Amer. Math. Monthly 80 (1973), 184-186.

Department of Mathematics, Tokyo Metropolitan University, Fukasawa, Setagaya, Tokyo 158, JAPAN 